\title{
Effet du stress hydrique osmotique sur la germination des graines chez les provenances de Cèdre du Liban (Cedrus Libani A. Rich.) d'origine Turque
}

\author{
Hüseyin Dirik \\ Université d’Istanbul, Faculté Forestière, 80895 Bahçeköy, Istanbul, Turquie
}

(Reçu le 7 juillet 1999; accepté le 28 septembre 1999)

\begin{abstract}
Résumé - On a étudié l'effet du stress hydrique osmotique sur la germination des graines chez les provenances de Cèdre du Liban. En utilisant le polyéthylène glycol (PEG-6000) pour préparer les niveaux différents de stress hydrique osmotique suivant les traitements envisagés $(0,-2,-4,-6$ bars) on a procédé à la comparaison expérimentale des facultés germinatives et des temps moyens de germination des graines de dix provenances. Les résultats essentiels obtenus sont les suivants : d'une façon générale, on n'a pas déterminé de différences importantes entre les provenances correspondant au temps moyen de germination. Le taux de germination moyen pour toutes les provenances diminue de $48 \%$ à -2 bars, de $61 \%$ à -4 bars, et de $75 \%$ à -6 bars par rapport au traitement témoin (0 bars). Des différences très nettes entre les provenances étudiées du point de vue des taux de germination relatif dans les niveaux différents de stress hydrique osmotique ont été mises en évidence. Bien que les données climatiques manquent, il y a des relations entre les facultés à germer des provenances étudiées face aux stress hydriques du substrat et les conditions écologiques de leurs milieux naturels. Il semble que ces différences entre les provenances procèdent de variations intraspécifiques produites sous les effets des sélections naturelles.
\end{abstract}

Cèdre du liban / provenance / germination / stress hydrique / polyéthylène glycol

\begin{abstract}
Effect of osmotic water stress on germination of Lebanon Cedar (Cedrus libani A. Rich.) seeds from the provenances of Turkey. Seeds from ten provenances of Cedrus libani were subjected to germination test on filter paper saturated with polyethylene glycol (PEG 6000) solutions made up to provide osmotic potentials of $0,-2,-4$ and -6 bars. Mean germination percent for all provenances decreased about $48 \%$ in -2 bars, $61 \%$ in -4 bars and $75 \%$ in -6 bars treatments compared to control ( 0 bar) treatment. There were significant differences among the relative germination percent of the provenances in different treatments. In spite of climatic data, strong correlations were found between germination capabilities under different stress levels and the ecological conditions in the natural habitats of the provenances. Differences between the germination capabilities of the provenances under different stress conditions were interpreted as an intraspesific heritable variation constituted by natural selection.
\end{abstract}

Lebanon cedar / provenance / germination / water stress / polyethylene glycol

\section{INTRODUCTION}

Le Cèdre du Liban est une espèce qui s'étend dans la région méditerranéenne orientale. Plus de $90 \%$ de l'aire actuelle est située en Turquie [4]. En dehors de cette aire principale, il est présent au Liban et en Syrie.

L'aire naturelle de Cèdre du Liban en Turquie est localisé d'une façon très morcelée sur la chaîne du Taurus.

* Correspondance et tirés à part

Tél. 902122261108 ; Fax. 902122261113 ; e-mail: hdirik@istanbul.edu.tr 
D'après les dernières données, la surface totale dans ce pays est environ de 100000 ha. Du point de vue écologique, indifférent au substrat, le Cèdre du Liban occupe les variantes très froides et localement extrêmement froides des bioclimats humide, subhumide et semi-aride (étages supra, montagnard et oroméditerranéen) [19]. Cette occupation, naturellement, correspond à une amplitude écologique très large chez cette espèce.

D'autre part, les recherches réalisées chez les espèces de Cèdre $[3,8]$ ont montré que le Cèdre du Liban est bien adapté à la sécheresse. Les résultats acquis sur les six premières années des expérimentations effectuées en région d'Anatolie centrale (au climat semi-aride) ont confirmé cette observation [6]. Ces résultats rendent très important le Cèdre du Liban pour les travaux de boisement et de reboisement par voie de semis direct ou de plantation faits en conditions semi-arides.

En effet, une des différences de comportement pour la résistance à la sécheresse des arbres forestiers peut apparaître dans les facultés à germer de leurs graines en conditions de stress hydrique élevé. Diverses études $[5,7,10]$ réalisées dans ce domaine ont montré que, généralement, les graines des espèces et même des provenances venant de zones sèches, peuvent germer plus facilement que les autres sur des milieux stressés. Le but du présent travail est d'étudier l'effet du stress hydrique osmotique sur la germination des graines chez les provenances de Cèdre du Liban d'origine turque. Il s'agit aussi de mettre en évidence d'éventuelles différences entre diverses provenances, et les relations entre les comportements germinatifs des provenances étudiées face au stress hydrique du substrat et les conditions écologiques de leurs milieux naturels, afin de contribuer à préciser la réaction à la sécheresse de cette espèce, au niveau infraspécifique.

\section{MATÉRIEL ET MÉTHODE}

L'expérience a porté sur les graines récoltées des dix provenances qui représentent, en général, l'aire naturelle de Cèdre du Liban en Turquie (figure 1). Le tableau I rend compte de quelques données sur ces provenances. Les cônes ont été récoltés au mois de novembre 1997 où la fructification a été très abondante dans tous les peuplements. Les récoltes et les traitements des graines ont été réalisés par l'Institut de Recherches des Graines et d'Amélioration des Arbres Forestiers (Ankara). Les graines obtenues ont été conservées en chambre froide pendant cinq mois $\left(+3{ }^{\circ} \mathrm{C}\right)$, leur teneur en eau étant alors d'environ $8 \%$. Au mois de mai 1998 elles ont été mises en stratification pendant 21 jours à $+4{ }^{\circ} \mathrm{C}$ en milieu humide pour lever leur dormance. Après avoir prélevé des graines, pour chaque provenance, on a déterminé

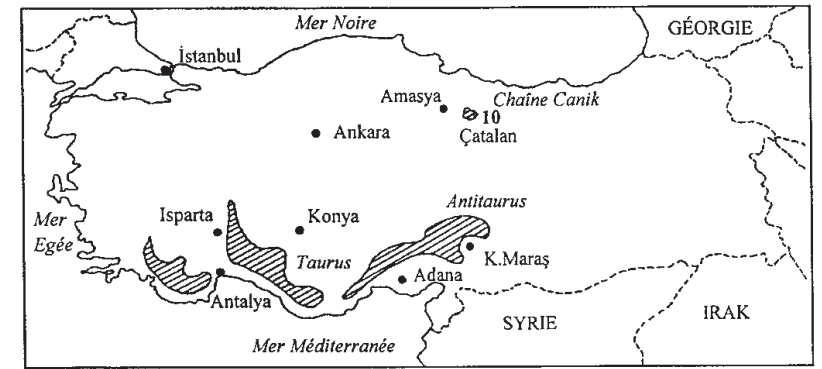

Aire schématique de Cèdre du Liban en Turquie

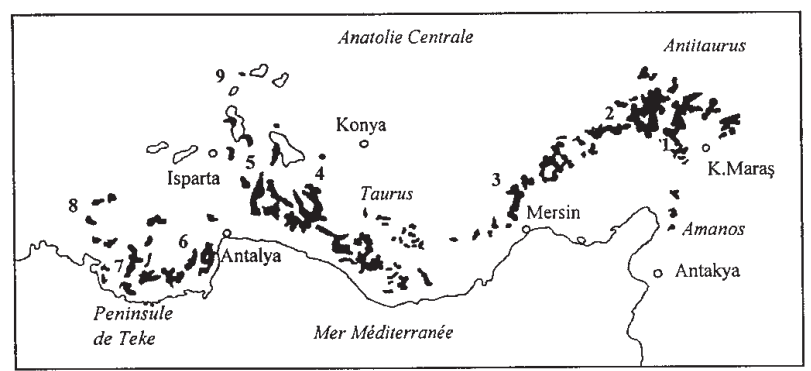

Localisation des provenances: $1:$ K.Maras-Andırn, 2: Adana-Kozan, 3:Mersin-Arslanköy, 4: Konya-Gökyurt, 5: Isparta-Kapıldă̆ı, 6: Antalya-Çı̆̆likara, 7: Antalya-Karaçay, 8: DeniziliBozdă̆, 9: Eskișehir-Sultandă̆ı, 10: Amasya-Çatalan

Figure 1. Aire naturelle de Cèdre du Liban en Turquie et localisation des dix provenances étudiées.

leurs facultés germinatives en faisant un essai de germination à l'aide de 5 répétitions de 50 graines pour chacune des 4 modalités de stress hydrique osmotique $(0,-2$, $-4,-6$ bars). Pour ajuster les niveaux envisagés de stress hydrique osmotique, on a utilisé le polyéthylène glycol (PEG-6000) suivant la protocole décrit par Michel et Kaufmann [14], et la méthode utilisée par Dunalp et Barnett [9]. Pour les essais de germination, les graines ont été mises à germer à la surface de deux lits de papiers saturés de solution PEG-6000 suivant les traitements envisagés et placés a l'intérieur de boîtes de Pétri $(11 \mathrm{~cm})$ transparentes, dans la chambre de germination. En conformité avec les normes ISTA [11], l'essai de germination a duré 21 jours avec des conditions de température de $20{ }^{\circ} \mathrm{C}$ et une hygrométrie de l'air saturée à $70-80 \%$.

Régulièrement, à intervalles de 2 jours, on a compté les graines qui ont germé et tous les substrats de papier ont été changés. La germination a été définie par l'apparition, puis le développement de la radicule.

\footnotetext{
${ }^{1}$ Temps moyen de germination: $T M G=\Sigma\left(n_{i} * t_{i}\right) / N$ où $n_{i}$ représente le nombre de graines germées le $t_{i}^{\mathrm{e}}$ jour après semis et $N$ le nombre total de graines germées [15].
} 
Tableau I. Caractéristiques des stations des provenances étudiées.

\begin{tabular}{|c|c|c|c|c|c|c|}
\hline No & Localité & Exposition & $\begin{array}{l}\text { Altitude } \\
\text { (m) }\end{array}$ & Longitude & Latitude & $\begin{array}{l}\text { Région } \\
\text { écologique }\end{array}$ \\
\hline 1 & $\begin{array}{c}\text { K.Maras } \\
\text { Andırın } \\
\text { Elmadağ }\end{array}$ & Ouest & 1500 & $36^{\circ} 28^{\prime} 08^{\prime \prime}$ & $37^{\circ} 37^{\prime} 03^{\prime \prime}$ & $\begin{array}{l}\text { arrière-pays } \\
\text { méditerranéen } \\
\text { (zonoécotone) }\end{array}$ \\
\hline 2 & $\begin{array}{l}\text { Adana } \\
\text { Kozan } \\
\text { Meydan }\end{array}$ & Nord-ouest & 1450 & $35^{\circ} 24^{\prime} 38^{\prime \prime}$ & $37^{\circ} 36^{\prime} 13^{\prime \prime}$ & $\begin{array}{l}\text { méditerranéen } \\
\text { supérieur } \\
\text { (orobiome) }\end{array}$ \\
\hline 3 & $\begin{array}{l}\text { Mersin } \\
\text { Arslanköy }\end{array}$ & Nord-ouest & 1600 & $34^{\circ} 14^{\prime} 00^{\prime \prime}$ & $37^{\circ} 00^{\prime} 00^{\prime \prime}$ & $\begin{array}{l}\text { méditerranéen } \\
\text { supérieur } \\
\text { (orobiome) }\end{array}$ \\
\hline 4 & $\begin{array}{c}\text { Konya } \\
\text { Gökyurt }\end{array}$ & Nord & 1500 & $32^{\circ} 02^{\prime} 00^{\prime \prime}$ & $37^{\circ} 49^{\prime} 39^{\prime \prime}$ & $\begin{array}{l}\text { Anatolie centrale } \\
\text { (zonobiome) }\end{array}$ \\
\hline 5 & $\begin{array}{c}\text { Isparta } \\
\text { Kapıdağ1 }\end{array}$ & Nord & 1600 & $30^{\circ} 42^{\prime} 20^{\prime \prime}$ & $38^{\circ} 05^{\prime} 03^{\prime \prime}$ & $\begin{array}{l}\text { arrière-pays } \\
\text { méditerranéen } \\
\text { (zonoécotone) }\end{array}$ \\
\hline 6 & $\begin{array}{l}\text { Antalya } \\
\text { Elmalı } \\
\text { Çığlıkara }\end{array}$ & Sud & 1850 & $29^{\circ} 48^{\prime} 00^{\prime \prime}$ & $36^{\circ} 33^{\prime} 25^{\prime \prime}$ & $\begin{array}{l}\text { arrière-pays } \\
\text { méditerranéen } \\
\text { (zonoécotone) }\end{array}$ \\
\hline 7 & $\begin{array}{l}\text { Antalya } \\
\text { Kaș } \\
\text { Karaçay }\end{array}$ & Nord & 1550 & $29^{\circ} 26^{\prime} 25^{\prime \prime}$ & $36^{\circ} 23^{\prime} 53^{\prime \prime}$ & $\begin{array}{l}\text { méditerranéen } \\
\text { supérieur } \\
\text { (orobiome) }\end{array}$ \\
\hline 8 & $\begin{array}{l}\text { Denizli } \\
\text { Acıpayam } \\
\text { Bozdağ }\end{array}$ & Nord-ouest & 1700 & $29^{\circ} 13^{\prime} 43^{\prime \prime}$ & $37^{\circ} 15^{\prime} 22^{\prime \prime}$ & $\begin{array}{l}\text { arrière-pays } \\
\text { méditerranéen } \\
\text { (zonoécotone) }\end{array}$ \\
\hline 9 & $\begin{array}{l}\text { Eskissehir } \\
\text { Afyon } \\
\text { Sultandağ1 }\end{array}$ & Nord-ouest & 1400 & $31^{\circ} 09^{\prime} 07^{\prime \prime}$ & $38^{\circ} 32^{\prime} 02^{\prime \prime}$ & $\begin{array}{l}\text { Anatolie centrale } \\
\text { (orobiome) }\end{array}$ \\
\hline 10 & $\begin{array}{l}\text { Amasya } \\
\text { Niksar } \\
\text { Çatalan }\end{array}$ & Sud & 1100 & $36^{\circ} 34^{\prime} 40^{\prime \prime}$ & $40^{\circ} 47^{\prime} 30^{\prime \prime}$ & $\begin{array}{l}\text { Chaine canik } \\
\text { (biome) }\end{array}$ \\
\hline
\end{tabular}

Avec les données obtenues, pour chaque provenance, on a calculé les temps moyens de germination ${ }^{1}$ et les taux de germination en fonction des 4 traitements. Pour comparer les valeurs de taux de germination des provenances étudiées on a appliqué la méthode d'analyse de variance factorielle simple du programme statistique ANOVA d'après deux variables (10 provenances et 4 traitements) et 5 répétitions. Le modèle statistique de cette méthode est $p_{i j k}=\mu+\alpha_{i}+\beta_{j}+\gamma_{k}+(\alpha \beta)_{i j}+(\alpha \gamma)_{i k}+(\beta \gamma)_{j k}+e_{i j k}^{2}$. En analyse de variance, on a utilisé les valeurs de germination relative $(p)$ pour chaque provenance (germination relative $=$ germination observée dans le traitement $x$ / germination dans le traitement temoin) afin d'éliminer les

\footnotetext{
${ }^{2} p_{i j k}: e$ valeur de germination relative, $\mu$ : moyenne générale, $\alpha_{i}$ : effet provenance, $\beta_{j}$ : effet traitement, $\gamma_{k}$ : effet de répétition, $(\alpha \beta)_{i j}$ : effet d'interaction provenance * traitement, $(\alpha \gamma)_{i k}$ : effet d'interaction provenance * répétition, $(\beta \gamma)_{j k}$ : effet d'interaction traitement $*$ répétition, $e_{i j k}:$ résiduelle.
}

effets des différences entre les valeurs des taux de germination dans le traitement témoin (0 bars). Avant analyse, ces valeurs ont été transformées avec arc $\sin p^{1 / 2}$. Finalement on a appliqué le test de Duncan pour mettre en évidence les différences entre les provenances et les traitements.

\section{RÉSULTATS}

Les résultats de l'essai de germination sont présentés dans le tableau II : taux moyens et temps moyens de germination. En ce qui concerne le temps moyen de germination, il n'y a pas de différences significatives entre les provenances étudiées. Cependant le temps de germination est légèrement plus élevé pour toutes les provenances dans les niveaux de stress hydrique élevés et plus particulièrement pour les provenances ayant le taux le plus bas 
Tableau II. Taux moyens et temps moyens de germination des provenances étudiées en fonction des traitements.

\begin{tabular}{|c|c|c|c|c|c|c|c|c|}
\hline \multirow[t]{3}{*}{ Provenances } & \multicolumn{8}{|c|}{ Taux moyens de germination (\%) et temps moyens de germination (jour) en fonction des traitements } \\
\hline & \multicolumn{2}{|c|}{ O bars } & \multicolumn{2}{|c|}{-2 bars } & \multicolumn{2}{|c|}{-4 bars } & \multicolumn{2}{|c|}{-6 bars } \\
\hline & $\%$ & jour & $\%$ & jour & $\%$ & jour & $\%$ & jour \\
\hline Andirın (1) & $\begin{array}{c}40,0 \\
( \pm 3,16)\end{array}$ & 11,4 & $\begin{array}{c}19,4 \\
( \pm 3,97)\end{array}$ & 11,8 & $\begin{array}{c}13,6 \\
( \pm 0,65)\end{array}$ & 12,2 & $\begin{array}{c}8,0 \\
( \pm 1,73)\end{array}$ & 13,5 \\
\hline Kozan (2) & $\begin{array}{c}49,2 \\
( \pm 4,15)\end{array}$ & 8,1 & $\begin{array}{c}25,2 \\
( \pm 4,15)\end{array}$ & 8,8 & $\begin{array}{c}21,8 \\
( \pm 2,49)\end{array}$ & 9,2 & $\begin{array}{c}12,0 \\
( \pm 2,00)\end{array}$ & 9,6 \\
\hline Arslanköy (3) & $\begin{array}{c}38,8 \\
( \pm 4,15)\end{array}$ & 10,2 & $\begin{array}{c}25,0 \\
( \pm 3,61)\end{array}$ & 10,7 & $\begin{array}{c}16,2 \\
( \pm 1,48)\end{array}$ & 9,4 & $\begin{array}{c}6,2 \\
( \pm 1,48)\end{array}$ & 13,4 \\
\hline Gökyurt (4) & $\begin{array}{c}46,2 \\
( \pm 3,63)\end{array}$ & 7,6 & $\begin{array}{c}25,8 \\
( \pm 3,49)\end{array}$ & 8,7 & $\begin{array}{c}20,0 \\
( \pm 2,45)\end{array}$ & 9,2 & $\begin{array}{c}21,8 \\
( \pm 2,68)\end{array}$ & 9,0 \\
\hline Kapıdağg 1 (5) & $\begin{array}{c}55,2 \\
( \pm 5,76)\end{array}$ & 7,8 & $\begin{array}{c}27,4 \\
( \pm 3,85)\end{array}$ & 9,0 & $\begin{array}{c}20,8 \\
( \pm 3,35)\end{array}$ & 9,8 & $\begin{array}{c}14,6 \\
( \pm 2,19)\end{array}$ & 9,9 \\
\hline Çıglıkara (6) & $\begin{array}{c}61,6 \\
( \pm 4,56)\end{array}$ & 9,4 & $\begin{array}{c}31,6 \\
( \pm 2,61)\end{array}$ & 10,6 & $\begin{array}{c}31,4 \\
( \pm 1,95)\end{array}$ & 10,6 & $\begin{array}{c}20,4 \\
( \pm 1,67)\end{array}$ & 11,8 \\
\hline Karaçay (7) & $\begin{array}{c}63,4 \\
( \pm 3,43)\end{array}$ & 10,4 & $\begin{array}{c}28,6 \\
( \pm 3,71)\end{array}$ & 10,3 & $\begin{array}{c}12,6 \\
( \pm 1,67)\end{array}$ & 12,4 & $\begin{array}{c}8,0 \\
( \pm 1,73)\end{array}$ & 12,1 \\
\hline Bozdag (8) & $\begin{array}{c}40,8 \\
( \pm 3,63)\end{array}$ & 8,9 & $\begin{array}{c}24,0 \\
( \pm 3,74)\end{array}$ & 10,0 & $\begin{array}{c}16,0 \\
( \pm 2,00)\end{array}$ & 10,9 & $\begin{array}{c}9,0 \\
( \pm 4,12)\end{array}$ & 11,1 \\
\hline Sultandağ 1 (9) & $\begin{array}{c}56,0 \\
( \pm 4,90)\end{array}$ & 9,1 & $\begin{array}{c}32,0 \\
( \pm 2,83)\end{array}$ & 10,3 & $\begin{array}{c}30,2 \\
( \pm 4,71)\end{array}$ & 11,1 & $\begin{array}{c}18,6 \\
( \pm 1,67)\end{array}$ & 10,8 \\
\hline Çatalan (10) & $\begin{array}{c}36,2 \\
( \pm 3,63)\end{array}$ & 8,2 & $\begin{array}{c}14,2 \\
( \pm 2,86)\end{array}$ & 9,8 & $\begin{array}{c}4,8 \\
( \pm 1,10)\end{array}$ & 12,0 & $\begin{array}{c}2,6 \\
( \pm 1,10)\end{array}$ & 13,8 \\
\hline
\end{tabular}

Les valeurs entre parenthèses montrent les écarts-types.

Tableau III. Analyse de variance pour la comparaison de taux de germinations relatives des dix provenances en fonction des quatre modalités de stress hydrique osmotique.

\begin{tabular}{|c|c|c|c|c|c|}
\hline \multirow{2}{*}{$\begin{array}{l}\text { Sources de variations } \\
\text { Effets principaux }\end{array}$} & \multirow{2}{*}{$\begin{array}{c}\text { Degrés de liberté } \\
16\end{array}$} & \multirow{2}{*}{$\begin{array}{c}\text { Sommes des carrés } \\
6124,570\end{array}$} & \multirow{2}{*}{$\begin{array}{c}\text { Carrés moyens } \\
382,786\end{array}$} & \multicolumn{2}{|c|}{$F_{\text {calc }}$} \\
\hline & & & & 237,007 & $* * *$ \\
\hline Provenance & 9 & 529,218 & 58,802 & 36,408 & $* * *$ \\
\hline Traitement & 3 & 5593,028 & 1864,343 & 1154,333 & $* * *$ \\
\hline Répétition & 4 & 2,324 & 0,581 & 0,360 & N.S. \\
\hline Interactions & 75 & 547,657 & 7,302 & 4,521 & $* * *$ \\
\hline Provenance $*$ traitement & 27 & 447,217 & 16,564 & 10,256 & $* * *$ \\
\hline Provenance $*$ répétition & 36 & 61,859 & 1,718 & 1,064 & N.S. \\
\hline Traitement $*$ répétition & 12 & 38,581 & 3,215 & 1,991 & $*$ \\
\hline Résiduelle & 108 & 174,429 & 1,615 & & \\
\hline Total & 199 & 6846,656 & 34,405 & & \\
\hline
\end{tabular}

*** : significant au niveau de 0,001 .

* : significant au niveau de 0,05 .

N.S. : non significant.

de germination (Çatalan, Karaçay, Andirin). Les résultats d'analyse de variance appliquée d'après les valeurs de taux de germination ont mis en évidence des différences statistiquement significatives $(0,001)$ entre les traite- ments, entre les provenances face aux différents niveaux de stress hydrique osmotique et aussi des significativités pour l'interaction de provenance * traitement $(0,001)$ et de traitement $*$ répétition $(0,05)($ tableau III). 
Tableau IV. Taux de germinations relatives des provenances étudiées en fonction des traitements, et leurs classements et groupements d'après les résultats d'analyse de variance factorielle et test de Duncan.

\begin{tabular}{lccccc}
\hline Provenances & \multicolumn{6}{c}{$\begin{array}{c}\text { Germination relative }(\%)=\text { germination } \\
\text { observée dans le traitement } x \text { / germination } \\
\text { dans le traitement témoin }\end{array}$} \\
\hline & 0 bars & -2 bars & -4 bars & -6 bars \\
\hline Çatalan (10) & 100 & 39,2 & 13,3 & 7,2 & $\mathrm{a}$ \\
Karaçay (7) & 100 & 45,1 & 19,9 & 12,6 & $\mathrm{ab}$ \\
Andırın (1) & 100 & 48,5 & 34,0 & 20,0 & $\mathrm{bc}$ \\
Arslanköy (3) & 100 & 64,4 & 41,8 & 15,9 & $\mathrm{bc}$ \\
Kapıdağ (5) & 100 & 49,6 & 37,7 & 26,4 & $\mathrm{bc}$ \\
Bozdağ (8) & 100 & 58,8 & 39,2 & 22,1 & $\mathrm{bc}$ \\
Kozan (2) & 100 & 51,2 & 44,3 & 24,4 & $\mathrm{bc}$ \\
Çığlıkara (6) & 100 & 51,3 & 51,0 & 33,1 & $\mathrm{c}$ \\
Sultandağ (9) & 100 & 57,1 & 53,9 & 33,2 & $\mathrm{c}$ \\
Gökyurt (4) & 100 & 55,8 & 43,3 & 47,2 & $\mathrm{c}$ \\
\hline
\end{tabular}

Chaque lettre montre un groupe de provenances statistiquement différentes au niveau de 0,05 selon le test de Duncan.

Selon le test de Duncan, la comparaison des 4 niveaux de traitements $(0,-2,-4,-6$ bars $)$ a montré que les traitements sont tous significativement différents au seuil statistique de 0,05. Quant à la comparaison des taux de germination des dix provenances en fonction des traitements, elle distingue trois catégories statistiquement différentes au seuil de significativité de 0,05 et les provenances se rangent comme sur le tableau IV.

Il est ainsi possible de les caractériser et d'essayer de les classer en fonction de leur résistance au stress hydrique osmotique du substrat en phase de germination. Les provenances de Gökyurt, de Sultandagi et de Çiglikara sont les trois provenances ayant les taux de germination les plus élevés au niveau des traitements -4 et -6 bars. À ces niveaux de stress hydrique osmotique les provenances ayant les valeurs les plus basses sont les provenances de Çatalan, de Karaçay et d'Andirin (figure 2).

\section{DISCUSSION}

La faculté germinative moyenne du traitement témoin ( 0 bars) est de $48,7 \%$. Selon les essais réalisés en Turquie par l'Institut de Recherches des Graines et d'Amelioration des Arbres Forestiers, le taux moyen de germination pour le Cèdre du Liban est de $50 \%$ en raison de la fragilité des graines et de l'existence de graines parasitées

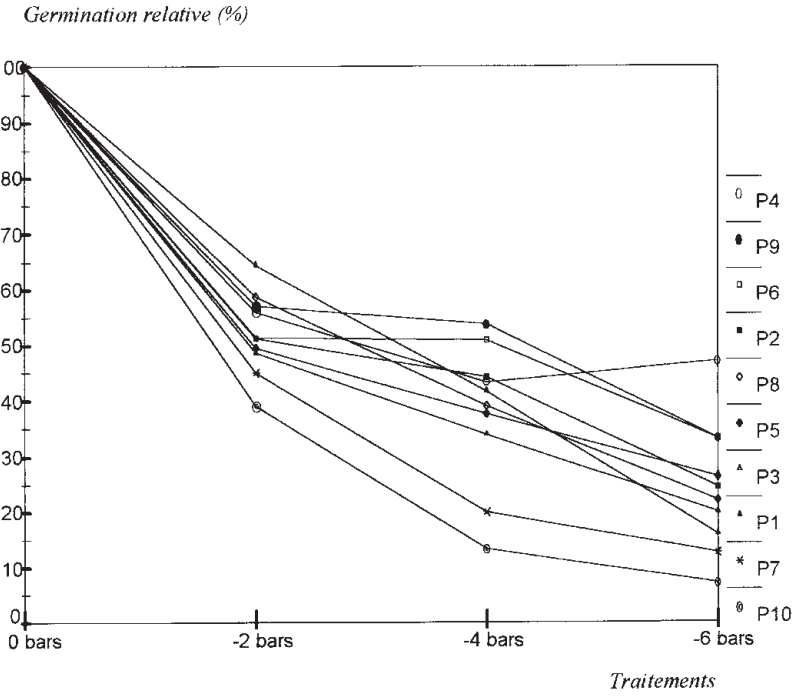

Figure 2. Répresentation graphique des valeurs de germination relative des traitements $-2,-4$ et -6 bars par rapport au temoin pour les provenances étudiées (P4: Gökyurt, P9 : Sultandağ1, P6 : Çığlıkara, P2 : Kozan, P8 : Bozdağ, P5 : Kapıdağı, P3 : Arslanköy, P1 : Andırın, P7 : Karaçay, P10 : Çatalan).

et vides, et aussi de leur faible pouvoir de conservation [16]. Toutefois la faculté germinative peut atteindre $70 \%$ pour des lots de bonne qualité $[1,17]$.

D'autre part, il y a des différences importantes de facultés germinatives pour le traitement témoin ( 0 bars) chez les provenances étudiées. On peut penser que ces différences résultent à la fois d'effets génétiques, des conditions des récoltes, et des traitements des graines qui peuvent varier selon les provenances.

En général le taux de germination diminue considérablement avec l'augmentation du stress hydrique osmotique du substrat. Des résultats similaires ont été obtenus par diverses études réalisées chez Pinus ponderosa et Pinus eldarica [7], chez Pinus halepensis, Pinus brutia et Pinus eldarica [5], chez Pinus halepensis [10] chez Pinus taeda [9] et chez Pinus halepensis et Pinus brutia [18]. D'aprés les résultats obtenus dans cette étude, le taux de germination diminue en moyenne de $48 \%$ à -2 bars, de $61 \%$ à -4 bars et de $75 \%$ à -6 bars par rapport au traitement témoin (0 bars).

Par ailleurs les résultats obtenus font apparaître, chez les provenances étudiées, des différences très nettes de faculté germinative dans chaque traitement. Cependant, selon les résultats d'analyse de variance, il s'agit des significativités pour les interactions. La diminution regulière des taux de germinations relatives en fonction des traitements et aussi des provenances rangées ont rendu 
vraisemblablement statistiquement significatives l'interaction de provenance * traitement. D'une façon générale, on voit qu'il y a une concordance entre les facultés germinatives des provenances étudiées vis-à-vis du stress hydrique osmotique du substrat et les conditions écologiques de leur milieu naturel.

Parmi les provenances étudiées, Çatalan a montré la germination relative la plus faible en condition de stress hydrique osmotique. Cette provenance vient du peuplement unique de Cèdre du Liban qui s'étend en zone d'Euxine (Euro-sibirienne). Cette région (Chaîne Canik) est sous l'effet des vents chargés d'humidité venant de la mer Noire. Les précipitations tombent en toutes saisons et le climat est de type humide [2]. D'autre part le peuplement de Çatalan, avec le peuplement d'Akincilar, sont considérés comme de véritables reliques témoignant de l'extension de la cédraie antique. Etant situés $300 \mathrm{~km}$ au nord des peuplements méditerranéens, leur écologie est très différente de celle qui caractérise le reste de l'aire naturelle [1]. Sa germination faible peut être due également à l'étroitesse de sa base génétique en raison de sa situation isolée. Viennent ensuite les provenances de Karaçay et d'Andirin. Karaçay est situé au sud de la Péninsule de Teke dans la région du Taurus occidental (figure 1). D'après les données climatiques, la Péninsule de Teke est la partie la plus humide de cette région, ce qui a justifié sa séparation d'avec les régions voisines, pour le transfert des graines chez le Cèdre du Liban [2]. Quant à Andirin, cette provenance qui se trouve dans la région de l'Antitaurus, elle a une situation topographique ouverte aux vents chargés d'humidité venant de la Montagne d'Amanos. Comme en Montagne d'Amanos, les précipitations d'été et l'existence des plusieurs espèces faisant partie des groupements végétaux de la zone Euro-sibirienne, prouvent que les conditions écologiques y sont différentes de l'ensemble de la région de l'Antitaurus.

Parmi les provenances étudiées dans la cadre de cet essai, Gökyurt, Sultandagi et Çiglikara apparaissent comme les provenances les plus résistantes aux stress hydriques élevés du substrat de germination. Localement les cédraies de Gökyurt et de Sultandagi se trouvent sur les versants orientés vers l'Anatolie Centrale (orobiome d'Anatolie Centrale) dont le climat est sec et continental $[2,13]$. Gökyurt est une population isolée et a une situation proche des steppes d'Anatolie Centrale. À l'exception de Çatalan et d'Akincilar situées près de la Mer Noire, le peuplement de Sultandagi correspond à la limite nord de la cédraie turc. Avec le peuplement de Gökyurt, elle constitue une véritable relique anatolienne de la cédraie. Toutes deux méritent une attention toute particulière pour leur conservation [1]. Çiglikara se situe dans un bassin de dépression fermé aux influences marines dans la région du Taurus occidental. C'est pour- quoi, localement le climat de ce bassin est semi-aride et continental $[12,2]$. Toutefois, Çiglikara contient une des cédraies les plus belles de la Turquie et il y a quelques « arbres monuments » ayant approximativement 2000 ans.

S'il existe donc, dans les grandes lignes, des relations entre les germinations relatives des provenances étudiées et les conditions écologiques de leur milieu naturel, ces analyses sont étayées par les résultats des recherches écologiques réalisées sur le Cèdre du liban $[2,12,13]$. Elles sont cependant limitées en raison de l'insuffisance du nombre de stations météorologiques installées en cédraie. Des corrélations similaires ont été déterminées dans d'autres études réalisées chez Pinus halepensis, Pinus brutia et Pinus eldarica [5] et chez Pinus halepensis [10]. Il semble que ces différences entre les provenances résultent de variations héréditaires intraspécifiques. Selon les résultats des analyses effectuées sur le pollen et les fossiles [2], en Turquie les cédraies s'etendaient selon une diagonale depuis les régions d'Anatolie du sud, vers l'Anatolie centrale et du nord au cours d'une période humide (23000 à 17000 avant J.C.). Dès que l'aridité a commencé à dominer en Anatolie centrale, l'aire naturelle de cette espèce a reculé vers les montagnes du pourtour méditerranéen. Ainsi, étant des populations reliques les cédraies de Gökyurt et de Sultandagi en région semi-aride et la cédraie de Çatalan en région semi-humide sont restées isolées [2]. On peut donc penser que les fréquences des gènes qui contrôlent la résistance au stress hydrique du substrat au cours de la germination des graines ont augmenté sous les effets de la sélection naturelle, de génération en génération, et qu'ainsi les populations génétiquement différenciées et qui peuvent se régénérer naturellement en conditions sèches se sont maintenues (Gökyurt, Sultandagi).

Enfin, pour expliquer l'adaptation à la sécheresse d'une espèce il est nécessaire d'étudier en détail d'autres caractéristiques que celles qui prévalent au moment de la germination. Cependant les résultats de l'étude entreprise ici pourront contribuer à ce sujet chez le Cèdre du Liban, la réussite de la germination étant déterminante dans la pérennité de peuplements régénérés naturellement.

\section{RÉFÉRENCES}

[1] Alptekin C.Ü., Bariteau M., Fabre J.P., Le Cèdre de Turquie : Aire naturelle, insectes ravageurs, perspectives d'utilisation pour les reboisements en France, Rev. For. Fr. XLIX-1 (1997) 19-31.

[2] Atalay I., Sedir (Cedrus libani A. Rich.) Ormanlarinin Yayilis Gösterdigi Alanlar ve Yakin Çevresinin Genel Ekolojik Özellikleri ile Sedir Tohum Transfer Rejyonlanmasi (General Ecological Properties of Natural Occurence Areas of Cedar 
(Cedrus libani A. Rich.) Forests and Regioning of Seed Transfer of Cedar in Turkey) OGM Yayin 663, Ankara, 1987.

[3] Bariteau M., Ferrandes P., l'Amélioration des Cèdre en France, Symposium International sur le Cèdre (22-27 Oct.1990Antalya) Institut de Recherche Forestière Publications Diverses 59 (1990) 366-378.

[4] Bariteau M., Ferrandes P., Les Cèdres, in : Gallais A., Bannerot H., L'Amélioration des plantes, Ed. INRA Paris, 1992, pp. 732-743.

[5] Calamassi R., Falusi M., Tocci A., Variazione geografica e resistenza a stress idrici in semi di Pinus halepensis Mill., Pinus brutia Ten. E Pinus eldarica Medw. Ann. Ist. Sper. Selv. XI (1980) 195-230.

[6] Dagdas S., Iç Anadolu Bölgesinde kurulu Sedir orijin denemelerinin ilk alti yillik sonuçlari (Variation in growth of six-year old provenance test of Cedar (Cedrus libani A. Rich.) established in central Anatolia), Iç An. Orm. Arst. Ens. Yay. 79 (1995) 117-136.

[7] Djavanshir K., Reid C.P.P., Effect of moisture stress on germination and radicle development of Pinus eldarica Medw. and Pinus ponderosa Laws, Can. J. For. Res. 5 (1975) 80-83.

[8] Ducrey M., Adaptation du Cèdre de l'Atlas (Cedrus atlantica Manetti) au climat méditerranéen : Aspects écophysiologiques de sa réaction à la sécheresse, in. M'Hirit O., Samih A., Malagnoux M. (Eds.). Le Cèdre de l'Atlas, Ann. Rech. For. Maroc. 27, 1 (1993) 140-153.

[9] Dunalp J.R., Barnett J.P., Manipulating loblolly pine (Pinus taeda L.) seed germination with simulated moisture and temperature stress, in : Duryea M.L., Brown G.N (Eds.). Seedling physiology and reforestation succes, Martinus Nijhoft/Dr. W Junk Publishers, Dordrecht, 1984, pp. 61-73.

[10] Falusi M., Calamassi R., Tocci A., Sensitivity of seed germination and seedling root growth to moisture stress in four provenances of Pinus halepensis Mill., Silvae Genet. 32, 1-2 (1983) 4-9.

[11] ISTA., International rules for seed testing. Annexes 1976, Seed Sci. Technol. 4 (1976) 51-177.

[12] Kantarci M.D., Türkiye sedirleri (Cedrus libani A.Richard) ve dogal yayilis alaninda bazi ekolojik iliskiler (Zeder (Cedrus libani A. Richard) in der Türkei un manche ökologische verhältnisse in ihrer verbreitungsgebiet), I.Ü. Orman Fak. Der. (A) 32, 2 (1982) 113-198.

[13] Kantarci M.D., Türkiye'de sedir ormanlarinin yayilis alaninda ekolojik iliskiler (The ecological interrelations at the cedar forest distribution areas in Turkey). Uluslararasi Sedir Sempozyumu (22-27 Ekim 1990-Antalya). Ormancilik Arastirma Enstitüsü Muhtelif Yayinlar 59, 1990, pp. 12-25.

[14] Michel B.E., Kaufmann M.R., The osmotic potential of polyethylene glycol 6000, Plant. Physiol. 51 (1973) 914-916.

[15] Müller C., Bonnet-Masimbert M., Amélioration de la germination des faînes (Fagus silvatica) par prétraitement en presence de polyéthylène glycol, Ann. Sci. For. 40, 2 (1983) 157-164.

[16] OAE., Önemli bazi agaç türlerinde tohum özellikleri, saklanma sartlari ve çimlenme engelinin giderilmesi islemleri, OAE. Arast. Bült. 11 (1980) 2.

[17] Odabasi T., Lübnan Sediri (Cedrus libani A. Rich.) nin Kozalak ve Tohumu Üzerine Arastirmalar (Recherches sur les Graines et les Cônes de Cèdre du Liban) O.G.M., Ankara, 1990.

[18] Thanos C.A., Skordilis A., The effects of light, temperature and osmotic stress on the germination of Pinus halepensis and P. brutia seeds, Seed Sci. Technol. 15 (1987) 163-174.

[19] Quezel P., La région méditerranéenne française et ses essences forestières, signification écologique dans le contexte circum-méditerranéen, Forêt Méd. 1, 1 (1979) 7-18. 